\title{
ON ORBIT DETERMINATION ACCURACY OF SPACE-VLBI SATELLITES
}

\author{
T. Borza, ${ }^{*}$ I. Fejes* and B. A. C. Ambrosius** \\ "FÖMI Satellite Geodetic Observatory, Penc, Budapest, Hungary \\ **Delft University of Technology, Faculty of Aerospace Engineering, \\ Kluyverweg 1, 2629 HS Delft, The Netherlands
}

ABSTRACT

The usefulness of space-VLBI for astrometric and geodynamic applications depends strongly on the orbit determination accuracy of the satellites involved. The requirements are very strict. In this paper, the results are presented of some preliminary investigations on the achievable orbit accuracy of the Soviet Radioastron and the ESA QUASAT space-VLBI satellites. For this purpose, simulations have been performed for a total of 23 orbital arcs, in which satellite observations from Precise Range and Range-rate Equipment (PRARE) were considered, as well as VLBl-type tracking of the satellite signals. The assumed tracking network consisted of 2 to 3 VLBI and 3 to 18 PRARE stations. The computations were carried out at Delft University of Technology using a computer program for covariance analysis of orbit determination problems.

\section{INTRODUCTION}

Two dedicated space-VLBI projects are in preparation for the years 1993-95. One, called Radioastron, is an approved and funded mission included in the space program of the Soviet Union. The other, called QUASAT, is being proposed in Western Europe and the USA as a joint ESA/NASA project. It is now under phase A study by ESA.

Space-VLBI has some very interesting potential applications in the field of astrometry and geodynamics (Reid 1984, Fejes et al 1986). Both applications need very precise orbit determination of the space antenna. For the Soviet Radioastron mission also real-time data processing is anticipated. This requires very accurate predictions of the position and the attitude of the satellite.

For these reasons it was decided to investigate the potential orbit determination accuracy of space-VLBI satellites in more detail. Earlier estimates for the orbit determination accuracy of QUASAT were reported by NASA and ESA study Teams (1984) and Jacobi et al (1985).

\section{ORBIT ACCURACY COMPUTATIONS}

The purpose of this study was to investigate how accurately the position of space-VLBI satellites can be determined and how well their orbits can be modeled. The first part of this work was carried out at Delft University of Technology in the Netherlands. For the computations, the ORAN error analysis program, developed by EG G/Washington Analytical Services Center (1978), was used. ORAN does not actually process observations, but it performs a covariance analysis of the orbit parameter estimation process, yielding indications for the accuracy of the orbit. It simulates a data reduction of real measurements of a given accuracy, while also considering errors in the measurement models and the dynamic models describing the motion of the satellite.

In general, the orbit determination accuracy depends on the accuracy of the measurement and dynamic models used in the orbit computations, the accuracy and spatial distribution of the tracking data and the length of the orbital are: For very short arcs the measurement parameters, such as the errors of the assumed station coordinates, may be the dominant error source, while the effects of dynamic parameters, such as the solar radiation pressure, will become dominant for longer arcs (Wakker et al 1983). To limit the effects of uncertainties in the solar radiation reflection properties of space-VLBi satellites, which may become quite large due to the very high area-to-mass ratios of such satellites, the orbital arcs considered had a maximum length of 24 hours. 


\section{Orbit configurations and ground stations}

Because the nominal orbits of both Radioastron and QUASAT are very eccentric, the visibility of the satellites is highly dependent of the orientation of the orbit relative to the ground stations. Therefore, a few variants of the nominal orbits were considered in which the epoch time and some of the orbit parameters were changed. Table 1 lists the epoch times and the full sets of orbital elements for five different configurations for which the simulations were performed. For Radioastron only two different cases (No. 1 and 2) were studied which differ in epoch time and in mean anomaly. For QUASAT the nominal (No. 3) set of orbit parameters was modified in order to optimize the tracking conditions for both the US and the European (Soviet) tracking stations (configuration No. 5). In Figure 1 the resulting ground tracks for the configurations 1,3 and 5 have been plotted. The satellite positions are marked at 1-hour intervals.

IABLE 1 The orbital parameters for various orbit configurations of Radioastron and QUASAT

\begin{tabular}{|c|c|c|c|c|c|}
\hline $\begin{array}{l}\text { Sat. } \\
\text { orb. conf. }\end{array}$ & $\begin{array}{c}\text { Radioast. } \\
\text { No. } 1 .\end{array}$ & $\begin{array}{c}\text { Radioast. } \\
\text { No. } 2 .\end{array}$ & $\begin{array}{c}\text { QUASAT } \\
\text { No. } 3 .\end{array}$ & $\begin{array}{c}\text { QUASAT } \\
\text { No. } 4 .\end{array}$ & $\begin{array}{c}\text { GUASAT } \\
\text { No. } 5 .\end{array}$ \\
\hline \multirow[t]{2}{*}{ Epoch } & 92.01 .01 & 92.01 .01 & 92.01 .01 & 92.01 .01 & 92.01 .01 \\
\hline & on om os & Th om os & on om os & on om os & $17 \mathrm{~h}$ om os \\
\hline$a[\mathrm{~km}]$ & 42200 & 42200 & 19900 & 19900 & 25600 \\
\hline e & 0.802 & 0.802 & 0.427 & 0.427 & 0.427 \\
\hline $1[\mathrm{deg}]$ & 65 & 65 & 30 & 30 & 30 \\
\hline$\Omega[$ deg] & 180 & 180 & 0 & 230 & 230 \\
\hline$\omega[$ deg] & 285 & 285 & 180 & 285 & 285 \\
\hline$M[$ deg] & 0 & 30 & 0 & 0 & 60 \\
\hline
\end{tabular}

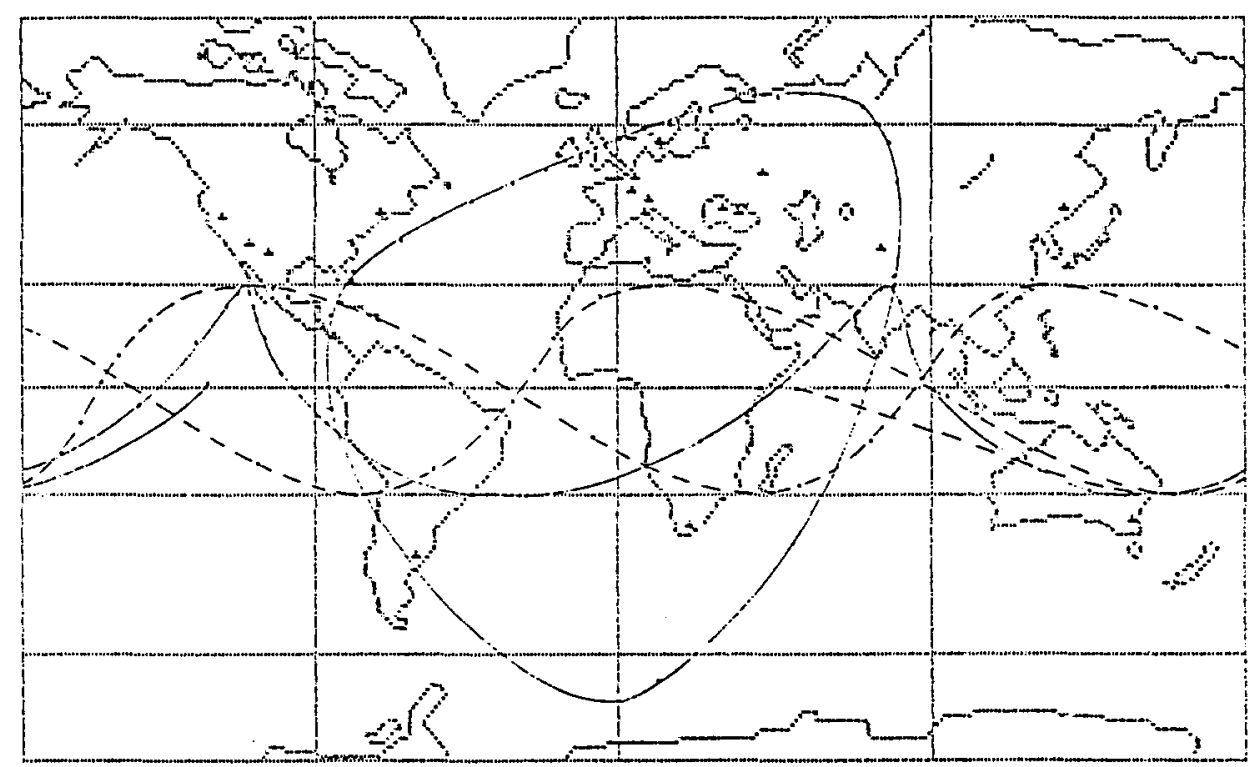

Fig. 1. The network of 18 PRARE tracking stations and some ground tracks of the satellites: Radioastron (No.1 orbital elements) QUASAT (No.3 orbital elements) $-\cdots-\cdots$ QUASAT (No. 5 orbital elements)

Also shown in Figure 1 are the locations of the assumed tracking stations. All these stations are radio-astronomical observatories and many of them are involved in VLBI work already. Basically, the overall network consists of a European, a Soviet and an American network, each comprising 5 stations all of which are in the Northern hemisphere. Therefore, three stations in Argentina, Australia and South-Africa were added to provide coverage from the Southern hemisphere. In the case of Radioastron, the southern tracking stations are of relatively minor importance, because the visibility periods ofthe satellite from these stations are only very brief. This is the results of the high eccentricity and the selected perigee angle, which causes Radioastron to spend most of its time at high altitude above the Northern hemisphere. The ground track plot in Figure 1 provides some indication of this effect. 


\section{The tracking techniques}

Potential tracking techniques for space-VLBI satellites have already been discussed in Fejes et al (1986). From a qualitative evaluation of the latest anticipated orbital elements of Radioastron and OUASAT some general conclusions regarding the precise tracking of space-VLBI satellites can be drawn. Basically, there are five different methods of tracking which may be applied, possibly in combination with each other. These methods are:

1. A two way conerent link of the VLBI telemetry and data acquisition station(s), to measure range and range rate with high precision.

2. The postprocessing of VLBI science data.

3. Application of an accurate microwave tracking system like the "Precise Range and Range-rate Equipment" (PRARE) (1983) being developed for the ESA ERS-1 mission.

4. VLBI type tracking of the transmissions of the satellite.

5. It might be benificial to have a micro-accelerometer on board in order to measure the non-gravitational perturbing forces acting on the satellite directly. Due to limitations of the software used in this study, only the Prare and VLBI type tracking could be simulated. Therefore, the effects of the application of the other methods on the orbit determination accuracy will be considered in a later study.

\section{The assumed error model}

The tracking parameters and the error model for the simulations are listed in Table 2. As can be seen, tracking networks comprising various numbers of stations were considered and also combinations of measurement types: In addition, the length of the data are was varied. Apart from the measurement errors themselves, many potential error sources can be identified. They were assigned an absolute or a fractional error which ORAN propagated into the adjusted orbit. As to the overall simulation approach the following remarks apply:

IABLE 2 Satellite, tracking and error parameters for the orbit determination simulation

\begin{tabular}{|c|c|c|}
\hline & & Radioastron \\
\hline SATZLITE & $\begin{array}{l}\text { Area/mass ratio }\left[\mathrm{m}^{2} / \mathrm{kg}\right] \\
\text { Solar reflectivity }\end{array}$ & $\begin{array}{l}0.08 \\
1.5\end{array}$ \\
\hline $\begin{array}{l}\text { TRACKING } \\
\text { NETWONK }\end{array}$ & $\begin{array}{r}\text { Number of stations (PRARE) } \\
\text { (VLRI) } \\
\text { (PRARE+VLII) }\end{array}$ & $\begin{array}{ccc}15,10,5,3 & 18,15,10,5 \\
3,2 & 3 & \\
3 & - & \end{array}$ \\
\hline OESERATIONS & $\begin{array}{l}\text { Arr length [hour] } \\
\text { Type } \\
\text { Repetition rate } \\
\text { Cut-off elevation }\end{array}$ & $\begin{array}{l}24,14,11,7,4 \quad 24,13,5,3 \\
\text { range, range rate (PRARE), range(VISI) } \\
1 \text { measurement per } 5 \mathrm{~min} \\
\text { 20 [de6] }\end{array}$ \\
\hline 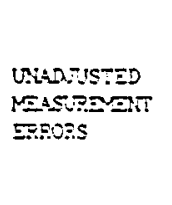 & $\begin{array}{l}\text { Station coordinates (PRARE) } \\
\text { (VLBI) } \\
\text { Fole position } \\
\text { Tida! uplift } \\
\text { Measure-ent noise } \\
\text { Tropospheric refraction } \\
\text { Ionospheric refraction }\end{array}$ & $\begin{array}{c}50 \mathrm{~cm} 10 \mathrm{~cm} \text { in earh local } \mathrm{xz} \text { comp. } \\
10 \mathrm{~cm}-"- \\
2 \mathrm{mas} \\
10 \% \\
\text { range } 5 \mathrm{~cm} \text { rangerate } 0.05 \mathrm{~cm} / \mathrm{s} \\
2 \% \\
2 \% \text { at } 8 \mathrm{grz}\end{array}$ \\
\hline $\begin{array}{l}\text { ULARTUSTED } \\
\text { DYNAMIC } \\
\text { ERRORS }\end{array}$ & $\begin{array}{l}\text { Gravity tleld } \\
\text { Gravitational parameter } \\
\text { Direce solar rallasion } \\
\text { Albedo radiation }\end{array}$ & $\begin{array}{l}10 \% \text { of differences between GSix: } 22 \text { and } \\
\text { GDM TL, truncated order, degree } 10 \times 10 \\
2.5 \times 10^{-10} \\
20 \%, 5 \% \\
50 \%\end{array}$ \\
\hline
\end{tabular}

- The assumed tracking data rate was 1 measurement in 5 minutes. Since the tracking periods are rather long, this rate yields sufficient observations per pass. A higher rate would note provide more information. It would only have an effect on the data-noise contribution to the errors inversely proportional to the square-root of the rate increase,

- The cut-of $f$ elevation was $20 \mathrm{deg}$, which is a rather strict condition,

- the 2 mas errors in polar motion and the $10 \%$ error level for the tidal forces are considered to be a fair estimates,

- two values, $50 \mathrm{~cm}$ and $10 \mathrm{~cm}$, were considered for the postition accuracy of the PRARE' stations, and $10 \mathrm{~cm}$ for the VLBI stations,

- the measurement noise for the PRARE ranges and the VLBI range-differences was assumed to be $5 \mathrm{~cm}$ and $0.5 \mathrm{~mm} / \mathrm{s}$ for range-rate,

- since PRARE is a dual frequency system, the ionospheric propagation effects can be largely

eliminated. The residual errors were estimated to amount to $2 \%$ of the effect at $8 \mathrm{GHz}$,

- the tropospheric refraction error was taken to be $2 \%$ of the effect,

- to simulate the uncertainties in a gravity field model that will be used in the actual orbit determination of space-VLBI satellites, an error model was used consisting of $10 \%$ of the differences between the GRIM-L2 and GEM-T1 models, truncated at degree and order 10 , - a $0.0025 \mathrm{ppm}$ error in the gravitational parameter was assumed which corresponds to its current estimated accuracy, 
- the effect of atmospheric drag was neglected because of the high altitude of the satellites, - due to the large area-to-mass ratio of space-VLBI satellites, the effect of solar radiation pressure is quite large. This means that errors in the modeling of this effect will also lead to large orbit errors. To investigate this, simulations for both a pessimistic $20 \%$, and an optimistic $5 \%$ fractional model error were performed,

- the Earth's albedo model error was assumed to amount to $50 \%$ of the effect.

\section{Results}

In total, 23 different simulations were performed to find some answers to the following questions: - What is the average orbit accuracy along a full 24-hour arc, assuming realistic tracking conditions and model errors?

- What are the individual contributions of the various error model parameters on the orbit accuracy?

- What are the required conditions to achieve a $1 \mathrm{~m}$ orbit accuracy?

IABLE 3 Summary of the orbit determination simulation results for the various cases considered $\left(* \Omega=180^{\circ}\right)$

\begin{tabular}{|c|c|c|c|c|c|c|c|c|c|c|c|}
\hline 1 & 2 & 3 & 4 & 5 & $\epsilon$ & & & ? & 7 & & 8 \\
\hline \multirow{2}{*}{ Arc } & \multirow{2}{*}{$\begin{array}{l}\text { Length } \\
\text { of arc } \\
\text { [rar) }\end{array}$} & \multirow{2}{*}{$\begin{array}{c}\text { Start and stop } \\
\text { from the epoch } \\
\text { [min] }\end{array}$} & \multirow{2}{*}{$\begin{array}{l}\text { Tracking } \\
\text { stations } \\
\text { No }\end{array}$} & \multirow{2}{*}{$\begin{array}{c}\text { Network } \\
\text { efficiency } \\
{[\%]}\end{array}$} & \multicolumn{2}{|c|}{ Assurned error } & \multicolumn{4}{|c|}{ abit errors RS [m] } & \multirow{2}{*}{$\begin{array}{l}\text { orbit } \\
\operatorname{con}{ }^{2} 1 \\
\mathrm{Nb}\end{array}$} \\
\hline & & & & & $\begin{array}{c}\text { solrad. } \\
[\not]]\end{array}$ & $\begin{array}{c}\text { st. coor. } \\
{[\mathrm{m}]}\end{array}$ & solrad & $\begin{array}{l}\text { stat. } \\
\text { coord. }\end{array}$ & other & total & \\
\hline \multicolumn{12}{|c|}{ OnIY PRARE tracking } \\
\hline 1 & 24 & $0-140$ & $1-15$ & 67 & 20 & 0.5 & 44.5 & 0.7 & - & 44.5 & 1 \\
\hline 2 & 24 & $0-1440$ & $6-10$ & 78 & 20 & 0.5 & 139.2 & 5.5 & 0.9 & 139.4 & 1 \\
\hline 3 & 14 & $110-945$ & $1-10$ & 93 & 20 & 0.5 & 19.0 & 4.5 & 0.9 & 19.5 & 1 \\
\hline 4 & 14 & $110-915$ & $6-10$ & 100 & 20 & 0.5 & 23.6 & 6.2 & 1.0 & 24.4 & 1 \\
\hline 5 & 7 & $110-530$ & $1-10$ & 87 & 20 & 0.5 & 3.8 & 6.9 & 1.2 & 8.0 & 1 \\
\hline 6 & 7 & $110-530$ & $6-10$ & 100 & 20 & 0.5 & 5.3 & 10.6 & 1.6 & 12.0 & 1 \\
\hline 7 & 24 & $0-1440$ & $1-18$ & 15 & 20 & 0.5 & 68.9 & 2.0 & 1.6 & 68.9 & 3 \\
\hline 8 & 24 & $0-1440$ & $1-18$ & 22 & 20 & 0.5 & 28.5 & 1.0 & 0.7 & 28.5 & 3* \\
\hline 9 & 13.3 & $1000-1800$ & $1-510-18$ & 28 & 20 & 0.5 & 22.5 & 1.7 & 0.6 & 22.6 & 4 \\
\hline 10 & 13.3 & $1000-1800$ & 2. $512-14$ & 37 & 20 & 0.5 & 76.2 & 3.5 & 1.5 & 76.3 & 4 \\
\hline 11 & 5.3 & $1000-1320$ & $1-5,11-15$ & 84 & 20 & 0.5 & 3.1 & 2.5 & 0.4 & 4.1 & 4 \\
\hline 12 & 5.3 & $1000-1320$ & 2. 5 12-14 & 81 & 20 & 0.5 & 4.3 & 4.9 & 0.5 & 6.6 & 4 \\
\hline 13 & 3.3 & $500-700$ & $1-5$ & 86 & 20 & 0.5 & 3.2 & 59.1 & 12.5 & 60.8 & 4 \\
\hline 14 & 3.3 & $500-700$ & $11-15$ & 99 & 20 & 0.5 & 3.7 & 6.0 & 0.8 & 7.4 & 4 \\
\hline 43 & 14 & $110-945$ & $6-10$ & 100 & 5 & 0.5 & 11.5 & 7.1 & 1.2 & 13.6 & 1 \\
\hline $6 a$ & 7 & $110-530$ & $6-10$ & 100 & 5 & 0.5 & 3.6 & 5.1 & 0.6 & 6.3 & 1 \\
\hline 15 & 11 & $0-660$ & 2. 9, 10 & 93 & 5 & 0.5 & 12.4 & 6.2 & 1.0 & 13.9 & 2 \\
\hline 16 & 7 & $0-420$ & 2. 910 & 100 & 5 & 0.5 & 5.2 & 10.8 & 0.9 & 12.0 & 2 \\
\hline $16 a$ & 7 & $0-420$ & द. 9,10 & 100 & 5 & 0.1 & 4.1 & 1.8 & 1.7 & 4.8 & 2 \\
\hline 17 & 4 & $0-240$ & 2, 9,10 & 100 & 5 & 0.5 & 2.8 & 22.2 & 1.0 & 22.4 & 2 \\
\hline 18 & 3.3 & $1000-1200$ & $11-15$ & 99 & 5 & 0.5 & 4.2 & 7.9 & 1.5 & 9.1 & 4 \\
\hline 23 & 4 & $60-300$ & $11-15$ & 100 & 5 & 0.5 & 4.4 & 7.1 & 1.2 & 8.4 & 5 \\
\hline \multicolumn{12}{|c|}{ On Iy VLBI tracking } \\
\hline 199 & 5.7 & $2 \infty 0-6 \infty$ & 2. 9,10 & 100 & 20 & 0.1 & 132.9 & 10.8 & 7.6 & 133.6 & 1 \\
\hline $15 a$ & 11 & $0-660$ & 2, 9,10 & 93 & 5 & 0.1 & 6.2 & 4.3 & $0.9 \mid$ & 7.6 & 2 \\
\hline 160 & 7 & $0-420$ & 2, 9,10 & 100 & 5 & 0.1 & 5.3 & 6.2 & 1.0 & 8.2 & 2 \\
\hline $17 a$ & 4 & $0-240$ & 2. 9, 10 & 100 & 5 & 0.1 & 4.8 & 9.4 & 1.2 & 10.6 & 2 \\
\hline 20 & 7 & $0-420$ & 9,10 & 100 & 5 & 0.1 & - & - & - & 33.5 & 2 \\
\hline 21 & 3.3 & $1000-1200$ & $11,13,14$ & 100 & 5 & 0.5 & 3.6 & 4.1 & 0.9 & 5.5 & 4 \\
\hline $21 a$ & 3.3 & $1000-1200$ & $11,13,14$ & 100 & 5 & 0.1 & 2.4 & 1.1 & 0.4 & 2.7 & 4 \\
\hline \multicolumn{12}{|c|}{ PRARE and VLBI trackIng } \\
\hline 22 & 7 & $0-$ & 2. 9. 10 & 100 & 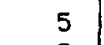 & 0.5 & 1.1 & 1.2 & 0.4 & 1.7 & 2 \\
\hline 223 & 7 & $0-420$ & 2. 910 & 100 & 5 & 0.1 & 1.0 & 0.3 & 0.2 & 1.1 & 2 \\
\hline
\end{tabular}

The results are summarized in Table 3. The first column contains the serial number of the investigated orbital are. Those arcs that had indentical tracking, but for which only different error models were used are marked by the letters a or $b$. In the second column the arc length is given, while the third column identifies the start and stop time of the data periods relative to the epoch time. The fourth column specifies which tracking stations (numbers according to Table 2) provided the measurements for the simulation. In the fifth column the "network efficiency" is indicated, 
which is the total accumulated tracking time divided by the number of observing stations times the arc length. The network efficiency is $100 \%$ when the full orbital are is observed from all stations. Next, the sixth column shows which assumptions were made for the solar reflectivity model error and the accuracy of the station coordinates. The main results are presented as four items in column 7, showing the root-mean-square (rms) of the orbit errors over the arc length. The first two items represent the individual contributions of the solar reflectivity and station coordinate errors. In the "other" sub-column the combined effect of all other error sources is given (i.e. measurement noise, ionospheric and tropospheric refraction, albedo, polar motion, gravity field and tidal effects). These errors are generally negligible, compared with the errors due to solar reflectivity and station coordinates. Finally, the eighth and last column contains the orbit configuration number according to Table 1.

From Table 3, a number of conclusions can be drawn. First the PRARE tracking simulations will be discussed. The rms orbit errors represent some sort of average error over the whole arc length. In reality, these errors are a function of time. The difference between arc 1 and 2 is only the number of tracking stations. Arc 1 is observed by all of the northern hemisphere stations but arc 2 only from the five Soviet stations. In the latter case the orbital errors are three times larger than in the first case. This is primarily caused by the degraded tracking geometry of the reduced network, which is confined to a much smaller area. The accuracy of the orbit determination of Radioastron reduces from $45 \mathrm{~m}$ to $140 \mathrm{~m}$ when using exclusively Soviet stations and assuming a $20 \%$ solar reflectivity model error. The largest contribution again comes from this error source, but the unfavourable geometry is also reflected in much larger errors due to the tracking station coordinate uncertainties. Similar, conclusions can be drawn with respect to the number and geometrical distribution of the tracking stations from the results for arcs $3-4$ and 5-5 of Radioastron, as well as arcs $9-10,11-12$ and 13-14 of QUASAT. The errors of are 8 are about half of those of are 7 (QUASAT). The difference is due to the improved tracking coverage, primarily archieved by a change in the position of the ascending node. The argument of perigee is also a very important parameter, because it can be used to position the apogee of the orbit over the hemisphere where the majority of tracking stations is located for optimum visibility conditions (see Figure 1). Due to the orbital period of 24 hours, the geometrical configuration of Radioastron and the tracking stations is repeated daily, but for QUASAT this is not the case. Therefore, the QUASAT orbit determination accuracy will be a function of time, with a repeat period much longer than the orbital period. The effect of solar radiation pressure on a satellite orbit is commulative in time. Therefore, it causes smaller errors in shorter arcs than in longer ones (arcs 4-6 and 4a-6a). On the other hand, the effects of station coordinate errors become larger for the shorter arcs, especially if the tracking geometry is not optimal (arcs 13, 16, 17). In these cases their contributions become very significant relative to the effects of all other error sources (comoare arcs 16-16a, $21-21 a$ and $22-22 a)$. It is surprising that change of orbital errors is nonlinear in consequence of change of solar radiation appriory errors (arcs 4-4a, 6-6a). Our opinion a single error parameter can not characterize suitably the error of orbital arc. On the other hand during the adjustment the sources of errors are not separate totally from each other.

VLBI-type tracking of satellite signals was simulated for configurations involving 2 or 3 stations. In the simulations for Radioastron the following stations were used: Medicina, Bearlake and Ussurisk. The length of the orbital are being observed simultaneously from these stations, is about 11 hours. From Table 3 it can be seen that using a $20 \%$ solar reflectivity model error, the orbit errors become very large for the nominal orbit configuration of Radioastron (arc 19). The results appear to be much better for arcs $15 a, 16 b$ and $17 a$, which use the more optimistic $5 \%$ solar reflectivity error and also a slightly modified orbit configuration. Another important difference is that these arcs also observe the perigee region, where the satellite is at a relatively low altitude and therefore provides good geometry for VLBI-type tracking. If the orbit determination is based on VLBI-type tracking from only 2 stations the errors may become four times larger than when using 3 stations, as can be seen from the differences between the results for arc 20 and are $16 \mathrm{~b}$. Therefore, it may be concluded that VLBI-type tracking needs a minimum of 3 stations. Also, it is interesting to note that PRARE tracking gives better results than VLBI-type tracking, which follows from a comparison of the results for arc $16 \mathrm{a}$ and 16b. The explanation is that PRARE provides absolute measurements of both range and range-rate, while VLBI only generates range-difference observations. This mainly effects the contribution of the station coordinate errors. Finally, it was found (arcs 22 and 22a) that by combining the PRARE and VLBI-type observations of a three-station network, the orbit determination accuracy for a 7-hour arc of Radioastron may reach a level of 1 to $2 \mathrm{~m}$.

\section{CONCLUSIONS}

The dominant part of the orbital errors of space-VLBl satellites originates from errors in the solar reflectivity models. All other effects can be modeled so accurately that they only yield small or negligible errors.

A $5 \%$ error in the solar reflectivity model seems to be a reasonable estimate, because in real orbit determinations, a scaling factor will be solved for which absorbs the average error. Nevertheless, for such large satellites, a good model for the variation of the cross-sectional area as a function of the direction of the incident radiation, as well as a model for the reflectivity of the various parts of the satellite will be required. 
A micro-accelerometer on board could be an alternative for this, since it can measure these non-gravitational forces. Therefore, developments in this field should be monitored carefully. The tracking stations should be well distributed over the globe to provide the best tracking geometries. The investigations of the orbit determination accuracy of space-VLBI satellites will be continued. In particular it is planned to investigate the possibility to obtain precise orbits of the space-VLBI satellites from using the VLBI science data itself as tracking data, and to study the potential role of micro-accelerometer data.

\section{ACKNOWLEDGEMENTS}

The athours whish to give special thanks to K. F. Wakker of the Faculty of Aerospace Engineering and F. 1. I. Brouwer of the Faculty of Geodesy for their support of this study carried out at Delft University of Technology. T. B. Acknowledges the financial support of the Rijkscommissie der Geodesie in the Netherlands and the Hungarian Intercosmos Council.

\section{REFERENCES}

1. Fejes, I. Almár, J. Adám, Sz. Mihály 1986: Space-VLBI : Potential Application in Geodynamics. Adv. Space Res. Vol 6, No. 9, D. 205.

N. Jacobi, C. S. Christensen, M. C. Hanna 1985: Study of Navigation Accuracy for the Proposed QUASAT Mission. AIAA 23rd Aerospace Sciences Meeting, Jan. 14-17 Reno, Nevada.

NASA and ESA Study Teams 1984: QUASAT: technical aspects of the proposed mission. Proc. QUASAT Workshop, Gross Enzersdorf, Austria, 18-22 June. ESA SP 213. p. 23.

M. J. Reid: HO Masers and Distance Measurements: the Impact of QUASAT. Proc. QUASAT Workshop, Gross Enzersdorf, Austria, 18-22 June, ESA SP 213, p. 185.

K. F. Wakker, B. A. C. Ambrosius, L. Aardoom 1983: Precise Orbit Determination for ERS-1. European Space Agency contract report, Delft University of Technology.

Ph. Hartl, Ch. Reigber 1985: Precise Range and range-rate equipment for satellite tracking (PRARE). CSTG Bulletin No 8. Ed. DGFI München, Fed. Rep. Germany.

T. V. Martin and J. J. MCCarthy July 1978: ORAN descriptive summary. EG G/Washington Analytical Services Center, Riverdale. 